\title{
Decompressive craniectomy for stroke in Brazil
}

\section{Craniectomia descompressiva para acidente vascular cerebral no Brasil}

\section{Dear Editor,}

We read with interest the recent article "Decompressive craniotomy for the treatment of malignant infarction of the middle cerebral artery: mortality and outcomes" ${ }^{\prime 1}$. Decompressive craniectomy (DC) for stroke is a well-established procedure for saving lives, despite the potential risk of it leading to severe disability in some patients. Because most of the published data comes from developed countries, with different socio-cultural characteristics, the article by Bongiorni et al. is very welcome, adding new and useful information about the reality of the Brazilian health care system. The authors reported, in the 20 patients enrolled in their study, a mortality rate of $30 \%$ after a 30 -day follow-up.

However, we disagree with the authors when they state that theirs was "the first study that assessed the role of DC outcome in malignant MCA infarction in an academic hospital in Brazil”. Actually, in 2008, Fiorot Jr et al. analyzed 18 patients, obtaining a mortality rate of $55 \%$ in 90 days. They attributed this high mortality rate to a late decision for surgery ${ }^{2}$. Afterwards, in 2010, Matos et al. published another study with 21 patients who underwent a DC, with a lower mortality rate (14.2\%) during the 180 days of follow-up ${ }^{3}$. Finally, in 2016, Vital et al. ${ }^{4}$ presented the greater Brazilian case series, with 60 patients, showing significant differences in mortality rates in patients under, or above, 60 years of age ( $41 \%$ vs $67 \%, p=0.039$ ).

These conflicting differences in mortality rates (as well as in functional outcomes) in the different teaching hospitals reflect the heterogeneity of analysis, indications, protocols and study designs. However, they provide useful and important information for Brazilian doctors who manage strokes. Multicenter studies, with standardized protocols, may provide more consistent information regarding the benefits of DC in our country, as well as helping with the selection of patients who may have greater benefits from this life-saving procedure considering, not only mortality, but also quality of life of the survivors.

Pedro Tadao Hamamoto Filho ${ }^{1}$, Andrei Fernandes Joaquim $^{2}$, Rodrigo Bazan ${ }^{1}$, Marco Antônio Zanini ${ }^{1}$

\section{References}

1. Bongiorni GT, Hockmuller MCJ, Klein C, Antunes APCM. Decompressive craniotomy for the treatment of malignant infarction of the middle cerebral artery: mortality and outcomes. Arq Neuropsiquiatr. 2017;75(7):424-8. https://doi.org/10.1590/0004-282X20170053

2. Fiorot Jr JA, Silva GS, Cavalheiro S, Massaro AR. Use of decompressive craniectomy in the treatment of hemispheric infarction. Arq Neuropsiquiatr. 2008;66(2A):204-8. https://doi.org/10.1590/S0004-282X2008000200012
3. Matos JP, Joaquim AF, Almeida JPC, Albuquerque LAF, Silva EG, Marenco HÁ et al.. Decompressive craniectomy in massive cerebral infarction. Arq Neuropsiquiatr. 2010;68(3):339-45. http://dx.doi.org/10.1590/S0004-282X2010000300002

4. Vital RB, Hamamoto Filho PT, Luvizutto GJ, Ducati LG, Braga GB, Nunes HRC et al. Decompressive hemicraniectomy in a South American population: morbidity and outcome analysis. PLoS One. 2016;11:e0146747. https://doi.org/10.1371/journal.pone.0146747

\footnotetext{
${ }^{1}$ Universidade Estadual Paulista, Faculdade de Medicina de Botucatu, Departamento de Neurologia, Psicologia e Psiquiatria, São Paulo SP, Brasil; ${ }^{2}$ Universidade Estadual de Campinas, Faculdade de Ciências Médicas, Departamento de Neurologia, Campinas SP, Brasil. 